\section{Avaliação da qualidade das prescrições de antimicrobianos dispensadas em unidades públicas de saúde de Belo Horizonte, Minas Gerais, Brasil, 2002}

\author{
Quality assessment of antibiotic prescriptions \\ dispensed at public health units in Belo Horizonte, \\ Minas Gerais, Brazil, 2002
}

\author{
${ }^{1}$ Farmácia Distrital Centro \\ Sul, Secretaria Municipal \\ de Saúde de Belo Horizonte, \\ Belo Horizonte, Brasil. \\ 2 Faculdade de Farmácia, \\ Universidade Federal de \\ Minas Gerais, \\ Belo Horizonte, Brasil. \\ ${ }^{3}$ Instituto de Ciências \\ Exatas, Universidade \\ Federal de Minas Gerais, \\ Belo Horizonte, Brasil. \\ Correspondência \\ P. M. Abrantes \\ Farmácia Distrital Centro \\ Sul, Secretaria Municipal \\ de Saúde de Belo Horizonte. \\ Rua Pernambuco 237. \\ Belo Horizonte, $M G$ \\ 30130-150, Brasil. \\ patricma@pbh.gov.br \\ patriciabrantes@oi.com.br
}

\begin{abstract}
Overuse of antibiotics is a public health problem and has raised discussions concerning their rational use. This cross-sectional study focuses on the use of systemic antibiotics under the Belo Horizonte Municipal Health Department, Minas Gerais, Brazil, evaluating prescriptions dispensed in March 2002, based on WHO indicators for antibiotic use. On average, 20\% of prescriptions dispensed involved at least one systemic antibiotic, among which approximately $46 \%$ originated from health care facilities not belonging to the municipal system itself. Amoxicillin was the most frequently prescribed antibiotic, followed by benzathine penicillin. Recording of technical data (dose, intervals between doses, administration, and treatment period) varied from 23.6 to $99.6 \%$. Some $10 \%$ of prescriptions failed to specify the treatment period. The study showed the need for rules to allow quality improvement of antibiotic prescriptions, assuring the rational use of such medication by municipal health services.
\end{abstract}

Anti-Bacterial Agents; Drug Prescriptions; Primary Health Care
Patrícia de Magalhães Abrantes 1 Sérgia Maria Starling Magalhães 2 Francisco de Assis Acúrcio ${ }^{2}$ Emília Sakurai 3

\section{Introdução}

Os antibacterianos constituem um grupo de medicamentos amplamente prescritos em atenção primária, ocupando sempre um dos primeiros lugares entre as classes de medicamentos mais utilizadas 1,2. O seu uso desnecessário e abusivo, tanto na comunidade quanto no meio hospitalar, tem sido extensivamente descrito na literatura 1,2,3,4.

O uso maciço e inadequado de antibacterianos tem implicações no aumento das taxas de resistência microbiana, existindo uma relação direta entre o quantitativo de antimicrobianos usados e a incidência de resistência bacteriana 4 .

À parte as conseqüências médicas e ecológicas, existe ainda um custo social e pessoal do uso inadequado desses medicamentos, que envolve custos diretos de tratamentos, internações, dias não trabalhados, faltas escolares, invalidez e morte. Tais implicações justificam esforços empreendidos no sentido de conhecer e racionalizar a utilização de antimicrobianos.

Dentre as diversas estratégias na área da utilização e monitorização do uso de medicamentos, destacam-se os estudos de hábitos de prescrição médica. Os prescritores exercem um importante papel na promoção do uso racional de medicamentos, de modo que a análise de seus hábitos de prescrição proporciona o conhecimento de aspectos da qualidade da terapia. Conseqüentemente, permite identificar problemas, implantar 
medidas corretivas e educativas e avaliar o impacto da adoção dessas medidas 1 .

Os erros de prescrição de antimicrobianos podem ser classificados em quatro níveis ${ }^{3}$. Considera-se, no primeiro nível, a prescrição de antimicrobianos não efetivos ou não indicados para a infecção em questão. Em uma segunda categoria, enquadram-se os erros de seleção do antimicrobiano, privilegiando os de segunda eleição. O terceiro nível refere-se aos erros de duração de tratamento. Os relacionados à dosagem, ao intervalo entre doses e à via de administração do medicamento são considerados no quarto nível.

Diversos estudos têm documentado o uso inadequado de antimicrobianos em situações às quais estes não se aplicam, principalmente nas infecções de origem viral 5,6. Do mesmo modo, tem sido apontada na literatura a adoção, na atenção primária, de opções terapêuticas inadequadas, privilegiando fármacos de segunda linha ou reservados ao uso hospitalar 7 . No que se refere aos erros de dosagem e duração de tratamento, Vergeles-Blanca et al. 2 observaram, no meio hospitalar, que a posologia inadequada foi responsável pela maioria dos erros de prescrição dos antimicrobianos. Fijn et al. 3 observaram também que a inadequação dos dados posológicos e do tempo de tratamento contribuíram como fatores de não-adesão aos protocolos de antibacterianos preconizados para uso no meio hospitalar.

Em estudo da prescrição de alguns medicamentos traçadores pela Secretaria Municipal de Saúde de Belo Horizonte (SMSA/BH), Minas Gerais, Brasil, Acúrcio et al. 8 observaram ausência de informações essenciais para dispensação e uso adequado do medicamento, indicando problemas com a prescrição que podem levar à má utilização do medicamento e a custos adicionais de tratamento.

As deficiências no registro de informações na prescrição são responsáveis por grande parte dos erros de medicação. A análise de prescrição pode contribuir para uma avaliação preliminar da qualidade da terapia, na medida em que evidencia falhas que comprometem a adesão ao tratamento e favorecem o aparecimento de reações adversas e falhas terapêuticas, prejudicando todo o esforço realizado pelo serviço público de saúde para o provimento adequado de medicamentos 9 .

Os gastos com medicamentos na SMSA/BH, inclusive com antibacterianos, vêm aumentando gradativamente nos últimos anos. Em 2002, os antibacterianos consumiram $15,5 \%$ do orçamento de medicamentos da Assistência Farmacêutica Básica, e, no período de 2000 a 2002, foi a segunda classe terapêutica de maior gasto.

Considerando o impacto sanitário e econômico da utilização de antibacterianos e o papel da prescrição no perfil de utilização desses medicamentos na comunidade, este estudo tem como objetivo realizar uma análise da prescrição de antibacterianos sistêmicos dispensados em Unidades Básicas de Saúde (UBS) da Gerência de Saúde Centro Sul (GERSA-CS). Para tal, atémse às questões formais de preenchimento e aos aspectos posológicos e de tempo de tratamento, essenciais para o sucesso da antibioticoterapia.

\section{Métodos}

Foi realizado um estudo descritivo, transversal, com base nas cópias das receitas de antibacterianos de uso sistêmico aviadas e retidas em unidades de atenção primária da GERSA-CS da SMSA/BH em março de 2002.

O Município de Belo Horizonte tem, de acordo com o Censo 2000, população de 2.229.697 habitantes e ocupa uma área de $330,23 \mathrm{~km}^{2}$, dividida em nove regiões político-administrativas. Em cada administração regional, existe uma gerência de saúde (GERSA) que administra os serviços do seu território. A coordenação das gerências de saúde é de responsabilidade do nível central, representado pela SMSA/BH, a qual, em 2000, coordenava 160 unidades de saúde de atenção primária e secundária 10 .

A regional Centro Sul da SMSA/BH é constituída por 11 unidades de saúde de atenção primária (centros de saúde), duas unidades de saúde de atenção secundária (policlínicas), dois laboratórios de análises clínicas e um centro de treinamento e referência em doenças infecto-parasitárias, representando $11 \%$ do total de unidades assistenciais ambulatoriais do município ${ }^{3}$. Essa regional concentra uma população de 265.743 habitantes, representando 11,92\% da população de Belo Horizonte. Agrega em torno de $14 \%$ dos domicílios particulares permanentes e cerca de $20 \%$ dos domicílios em vilas e favelas do município, correspondendo a $18 \%$ da população neste último tipo de residência 10 .

As farmácias das UBS da SMSA/BH atendem integralmente todas as receitas de moradores de Belo Horizonte. Para moradores de outros municípios, atendem integralmente receitas de medicamentos de uso em doenças agudas, dentre os quais os antibacterianos, desde que originadas em unidades pertencentes ao Sistema Único de Saúde (SUS) de Belo Horizonte.

Foram aviadas, no período, 19.528 receitas, das quais 4.088 continham antibacterianos de uso sistêmico, classificados, segundo a Anatomical Therapeutic Chemical (ATC), nos grupos J01 (antibacterianos para uso sistêmico) e G04 (antisépticos urinários, antiinfecciosos) 11. A fim de 
se verificar se existem diferenças na qualidade das receitas emitidas nas unidades de saúde da SMSA/BH em relação aos outros locais, as prescrições foram classificadas, segundo a origem, em internas, aquelas originadas em unidades de saúde da SMSA/BH, e externas, originadas em outras unidades do SUS.

Determinou-se o tamanho da amostra considerando-se $50 \%$ de receitas com preenchimento adequado de todos os itens para os antibacterianos, 95\% de grau de confiança, erro máximo de $5 \%$ com correção para população finita e margem de $20 \%$ de perdas, resultando em uma amostragem estratificada e proporcional de $40 \%$ de todas as receitas atendidas nas unidades de saúde pesquisadas. Em virtude da aproximação feita para cada centro de saúde, a amostra determinada foi de 930 receitas internas e 787 externas selecionadas por sorteio aleatório simples.

Foram avaliados os seguintes indicadores: especialidade do prescritor, número médio de medicamentos por receita, número médio de antibacterianos por receita. Em relação aos antibacterianos, avaliaram-se: a prescrição pelo nome genérico, presença de apresentação, forma farmacêutica, via de administração, dose, intervalo entre doses e duração do tratamento. Para as três últimas variáveis, foi averiguada a adequação aos dados da literatura 12,13 para o tratamento das infecções mais comuns em atenção primária.

Os dados coletados foram organizados em um banco de dados criado no programa Epi Info 2000 (Centers for Disease Control and Prevention, Atlanta, Estados Unidos), o qual foi utilizado também para realizar as análises estatísticas. Para a análise dos dados, procedeu-se à distribuição de freqüência das variáveis estudadas. As comparaçõs entre as proporções observadas nos grupos foram analisadas utilizando-se o teste do qui-quadrado. $\mathrm{O}$ nível de significância considerado foi de 0,05 .

Este projeto foi submetido ao Comitê de Ética em Pesquisa da Universidade Federal de Minas Gerais, tendo sido aprovado em 10 de julho de 2002.

\section{Resultados}

No mês de março de 2002, foi dispensado um total de 19.528 receitas nas UBS da GERSA-CS. Em 20,9\% (4.088), estava prescrito pelo menos um antibacteriano de uso sistêmico. As receitas internas representaram $53,9 \%$ do total, e em $17,5 \%$ destas foi prescrito antibacteriano de uso sistêmico, ao passo que a prescrição desses fármacos ocorreu em $27 \%$ das receitas externas. Observam-se diferenças estatisticamente significantes entre as receitas internas e externas quanto ao percentual de antibacterianos de uso sistêmico prescritos $\left(\chi^{2}=245,89 ; \mathrm{p}=0,000\right)$.

Em relação à origem das receitas externas amostradas, os hospitais públicos foram os que contribuíram com o maior percentual de receitas (73,2\%), seguidos pelos planos de saúde e consultórios particulares (22,4\%).

No contexto da utilização de antibacterianos, a especialidade médica evidencia os grupos profissionais que mais prescrevem, assim como seus hábitos de prescrição, permitindo intervenções direcionadas. Na Tabela 1, são apresentadas as especialidades do prescritor para as receitas amostradas. Observam-se diferenças estatisticamente significantes entre os dois grupos estudados nas especialidades: clínico $\left(\chi^{2}=61,13 ; \mathrm{p}=\right.$ $0,000)$, generalista $\left(\chi^{2}=149,30 ; p=0,000\right)$, gineco-

Tabela 1

Especialidade do prescritor de acordo com a origem da receita. Belo Horizonte, Minas Gerais, Brasil, 2002.

\begin{tabular}{|c|c|c|c|c|}
\hline \multirow[t]{2}{*}{ Item } & \multicolumn{2}{|c|}{ Receita interna $(n=930)$} & \multicolumn{2}{|c|}{ Receita externa $(n=787)$} \\
\hline & $\mathrm{n}$ & $\%$ & $\mathbf{n}$ & $\%$ \\
\hline Pediatra & 296 & 31,8 & 249 & 31,6 \\
\hline Clínico * & 177 & 19,0 & 49 & 6,2 \\
\hline Generalista * & 163 & 17,5 & 1 & 0,1 \\
\hline Ginecologista * & 36 & 3,9 & 6 & 0,8 \\
\hline Dentista & 21 & 2,3 & 16 & 2,0 \\
\hline Outras * & 18 & 1,9 & 37 & 4,7 \\
\hline Informação não disponível & 217 & 23,3 & 415 & 52,7 \\
\hline Ilegível & 2 & 0,2 & 8 & 1,0 \\
\hline
\end{tabular}

* Associação estatisticamente significante : $p<0,05$.

Fonte: Gerência de Saúde Centro Sul, Secretaria Municipal de Saúde de Belo Horizonte, Minas Gerais, Brasil. 
logista $\left(\chi^{2}=17,25 ; \mathrm{p}=0,000\right)$ e outras $\left(\chi^{2}=10,51\right.$; $\mathrm{p}=0,001)$. Verifica-se, no caso das prescrições internas, que o generalista foi o terceiro maior prescritor $(17,5 \%)$, com percentual próximo ao da clínica médica. Ressalta-se o elevado percentual de informação não disponível para a especialidade do prescritor, parcialmente explicada pelo fato de essa informação não ser obrigatória no receituário ou no carimbo do prescritor.

Foi prescrito um total de 2.136 medicamentos nas receitas internas e 2.002 nas externas. $\mathrm{O}$ número médio de medicamentos por prescrição foi de 2,3 nas internas e 2,5 nas externas.

O maior percentual das receitas continha dois medicamentos (cerca de 40\%) enquanto aproximadamente $30 \%$ apresentaram três especialidades.

Foram prescritos 941 antibacterianos nas receitas internas e 797 nas externas. Onze receitas internas $(1,2 \%)$ e dez externas $(1,3 \%)$ apresentaram dois antibacterianos de uso sistêmico, sendo as combinações mais freqüentemente observadas a benzilpenicilina benzatina com a benzilpenicilina procaína/potássica, a amoxicilina com o sulfametoxazol/trimetoprim e a amoxiclina com a benzilpenicilina benzatina.

Foram prescritos 19 antibacterianos distintos. Os prescritos com maior freqüência foram a amoxicilina (49,7\% nas receitas internas e $66,3 \%$ nas externas), a benzilpenicilina benzatina ( $24 \%$ nas receitas internas e $5,9 \%$ nas externas), a associação sulfametoxazol/trimetoprim (12,1\% nas receitas internas e $11 \%$ nas externas) e a eritromicina $(6,2 \%$ nas receitas internas e $3,4 \%$ nas externas). Os três antibacterianos prescritos com maior frequência representaram mais de $80 \%$ do total. Observaram-se diferenças estatisticamente significantes entre os dois grupos de prescrições para o percentual de receitas do medicamento amoxicilina $\left(\chi^{2}=48,10 ; p=0,000\right)$ e benzilpenicilina benzatina $\left(\chi^{2}=106,94 ; p=0,000\right)$. Dentre os antibacterianos não padronizados, os prescritos com maior freqüência foram o norfloxacino, nas receitas internas, e a amoxicilina/ácido clavulânico, nas externas.

$\mathrm{Na}$ Tabela 2, verifica-se a presença do registro do medicamento pelo nome genérico, da forma farmacêutica, apresentação, via de administração, dose, intervalo entre doses e do número de dias de tratamento para os antibacterianos prescritos. Diferenças estatisticamente significantes entre os dois grupos de prescrições foram observadas nas variáveis: nome genérico $\left(\chi^{2}=90,41\right.$; $\mathrm{p}=0,000)$, forma farmacêutica $\left(\chi^{2}=647,39 ; \mathrm{p}=\right.$ $0,000)$, apresentação $\left(\chi^{2}=13,33 ; p=0,000\right)$, via de administração $\left(\chi^{2}=21,68 ; \mathrm{p}=0,000\right)$ e número de dias de tratamento $\left(\chi^{2}=90,80 ; \mathrm{p}=0,000\right)$. Nota-se que, com exceção da via de administração e intervalo entre doses, as receitas externas apresentaram maiores percentuais de registros dessas informações.

As receitas de benzilpenicilina benzatina contribuíram para o elevado índice de ausência da forma farmacêutica e do nome genérico, já que estes estiveram presentes em um percentual muito pequeno. Ressalta-se a ausência de tempo de tratamento em cerca de $10 \%$ das receitas internas e externas. Quando avaliados os mesmos itens para cada um dos antibacterianos mais prescritos, verifica-se que, para a amoxicilina e benzilpenicilina benzatina, os maiores registros de presença de dados foram para as variáveis do-

Tabela 2

Presença de nome genérico, forma farmacêutica, apresentação, via de administração, dose, intervalo e número de dias de tratamento para os antibacterianos de uso sistêmico segundo sua origem. Belo Horizonte, Minas Gerais, Brasil, 2002.

\begin{tabular}{|c|c|c|c|c|}
\hline \multirow[t]{2}{*}{ Item } & \multicolumn{2}{|c|}{ Receita interna $(n=941)$} & \multicolumn{2}{|c|}{ Receita externa $(n=797)$} \\
\hline & n & $\%$ & $\mathrm{n}$ & $\%$ \\
\hline Nome genérico * & 609 & 64,7 & 676 & 85,7 \\
\hline Forma farmacêutica * & 222 & 23,6 & 204 & 25,6 \\
\hline Apresentação * & 376 & 40,0 & 388 & 48,7 \\
\hline Via de administração * & 754 & 80,1 & 562 & 70,5 \\
\hline Dose & 937 & 99,6 & 792 & 99,4 \\
\hline Intervalo entre doses & 931 & 98,9 & 794 & 99,6 \\
\hline Número de dias de tratamento * & 831 & 89,3 & 714 & 89,6 \\
\hline
\end{tabular}

* Associação estatisticamente significante: $p<0,05$.

Fonte: Gerência de Saúde Centro Sul, Secretaria Municipal de Saúde de Belo Horizonte, Minas Gerais, Brasil. 
se e intervalo entre doses, e os menores foram para forma farmacêutica e nome genérico, conforme observado na Tabela 3 .

Com relação à dose, observa-se que a ausência deste item nas prescrições internas de eritromicina ficou aquém do observado para os outros antibacterianos. As prescrições de sulfametoxazol/trimetoprim apresentaram percentuais elevados de ausência de apresentação, chegando a cerca de $90 \%$

Para uma avaliação do intervalo posológico, considerou-se o preconizado na literatura de referência 12,13 para o tratamento das infecções mais comuns na atenção primária, a saber: amoxicilina $-8 / 8$ horas; benzilpenicilina benzatina - dose única ou intervalos de 3-4 semanas; sulfametoxazol/trimetoprim - 12/12 horas e eritromicina - 6/6 horas. Verificou-se a adoção de intervalos posológicos atípicos, como de 24/24 horas para a benzilpenicilina benzatina $(1,5 \%)$, de $6 / 6$ horas para amoxicilina (1,3\%), $8 / 8$ horas para sulfametoxazol/trimetoprim (5,4\%) e $12 / 12$ horas para eritromicina $(1,2 \%)$. Não foram pesquisadas situações clínicas específicas para justificar tais intervalos.

A ausência do tempo de tratamento foi observada com freqüência maior que $20 \%$ nas prescrições internas de eritromicina e nas externas de sulfametoxazol/trimetoprim.

Na Tabela 3, verifica-se que a duração de tratamento com maior freqüência foi de dez dias para amoxicilina, eritromicina e sulfametoxa- zol/trimetoprim. Ao se compararem os grupos de prescrições internas e externas, observaramse diferenças estatisticamente significantes entre os grupos para a amoxicilina no tempo de tratamento de 14 dias $\left(\chi^{2}=9,73 ; p=0,018\right)$. Verifica-se uma grande variação nos tempos de tratamento instituídos, com a adoção de períodos de tratamento atípicos como menores que sete dias e períodos de 11 e 13 dias de tratamento. Consideraram-se intervalos usuais aqueles preconizados pela literatura, a saber: 3-4 semanas para a benzilpenicilina benzatina e 7, 10 e 14 dias para os demais antibacterianos.

\section{Discussão}

Este estudo constitui uma primeira abordagem para verificar, por meio de indicadores, alguns aspectos relativos à qualidade da prescrição de antibacterianos na SMSA/BH, considerando que esses são recursos fundamentais na prática médica e de grande impacto em termos de saúde pública. Adicionalmente, o interesse por essa classe nessa Secretaria deve-se ao fato de os recursos destinados ao suprimento desses medicamentos serem significativamente altos dentro do orçamento da assistência farmacêutica da instituição. Assim, verificar as condições de prescrição dos antibacterianos pode constituir etapa fundamental para uma utilização mais racional dessa classe terapêutica.

Número de dias de tratamento para a amoxicilina, eritromicina e sulfametoxazol+trimetoprim segundo origem das receitas. Belo Horizonte, Minas Gerais, Brasil, 2002.

\begin{tabular}{|c|c|c|c|c|c|c|c|c|c|c|c|c|}
\hline \multirow[b]{3}{*}{ Número de dias } & \multicolumn{4}{|c|}{ Amoxicilina } & \multicolumn{4}{|c|}{ Eritromicina } & \multicolumn{4}{|c|}{ Sulfametoxazol + trimetoprim } \\
\hline & \multicolumn{2}{|c|}{$\begin{array}{l}\text { Receita interna } \\
\qquad(n=468)\end{array}$} & \multicolumn{2}{|c|}{$\begin{array}{l}\text { Receita externa } \\
\qquad(n=528)\end{array}$} & \multicolumn{2}{|c|}{$\begin{array}{l}\text { Receita interna } \\
\qquad(\mathrm{n}=58)\end{array}$} & \multicolumn{2}{|c|}{$\begin{array}{l}\text { Receita externa } \\
\qquad(n=27)\end{array}$} & \multicolumn{2}{|c|}{$\begin{array}{l}\text { Receita interna } \\
\qquad(\mathrm{n}=114)\end{array}$} & \multicolumn{2}{|c|}{$\begin{array}{l}\text { Receita externa } \\
\qquad(\mathrm{n}=88)\end{array}$} \\
\hline & $\mathrm{n}$ & $\%$ & n & $\%$ & $\mathbf{n}$ & $\%$ & $\mathrm{n}$ & $\%$ & $\mathrm{n}$ & $\%$ & $\mathrm{n}$ & $\%$ \\
\hline Até 6 & 12 & 2,5 & 18 & 3,4 & 12 & 20,6 & 0 & 0,0 & 5 & 4,4 & 3 & 3,4 \\
\hline 7 & 56 & 12,0 & 47 & 8,9 & 11 & 19,0 & 8 & 29,6 & 15 & 13,2 & 11 & 12,5 \\
\hline $8-9$ & 21 & 4,5 & 9 & 1,7 & 2 & 3,5 & 0 & 0,0 & 2 & 1,8 & 3 & 3,4 \\
\hline 10 & 265 & 56,6 & 312 & 59,1 & 19 & 32,8 & 14 & 51,9 & 69 & 60,4 & 36 & 41,0 \\
\hline $11-13$ & 12 & 2,6 & 9 & 1,7 & 1 & 1,7 & 1 & 3,7 & 2 & 1,8 & 1 & 1,1 \\
\hline 14 * & 27 & 5,8 & 60 & 11,4 & 0 & 0,0 & 1 & 3,7 & 2 & 1,8 & 3 & 3,4 \\
\hline $15-20$ & 23 & 4,9 & 26 & 4,9 & 1 & 1,7 & 0 & 0,0 & 10 & 8,7 & 6 & 6,8 \\
\hline 21 & 0 & 0,0 & 5 & 1,0 & 0 & 0,0 & 0 & 0,0 & 0 & 0,0 & 2 & 2,3 \\
\hline Mais que 21 & 1 & 0,2 & 0 & 0,0 & 0 & 0,0 & 0 & 0,0 & 1 & 0,9 & 0 & 0,0 \\
\hline \multicolumn{13}{|l|}{ Informação } \\
\hline não disponível & 51 & 10,9 & 42 & 8,0 & 12 & 20,7 & 3 & 11,1 & 8 & 7,0 & 23 & 26,1 \\
\hline
\end{tabular}

* Associação estatisticamente significante para a amoxicilina: $p<0,05$.

Fonte: Gerência de Saúde Centro Sul, Secretaria Municipal de Saúde de Belo Horizonte, Minas Gerais, Brasil. 
Observou-se que cerca de $20 \%$ das receitas atendidas pela GERSA-CS continham algum antibacteriano sistêmico. Quando comparados com os de outros estudos, observam-se grandes variabilidades nesses percentuais 1,14,15. Os resultados podem estar relacionados a fatores nosológicos locais e a características dos prescritores, dos serviços de saúde, da qualidade da assistência e da atenção à saúde.

Dentro da perspectiva de acesso ao medicamento, verificou-se que as receitas externas contendo antibacterianos corresponderam a quase a metade do total de receitas dispensadas. Este percentual é alto e representa um consumo de recursos consideráveis para o município, fato que já havia sido apontado anteriormente por Acúrcio et al. ${ }^{8}$ para medicamentos de outras classes farmacológicas. Os planos de saúde e consultórios particulares foram a segunda origem mais freqüente de prescrições externas, o que suscita discussões sobre o papel das seguradoras na cobertura dos tratamentos médicos. $\mathrm{O}$ percentual de receitas externas contendo antibacterianos foi maior que o de internas, reforçando o papel do serviço público de saúde como provedor dessa classe de medicamentos para a clientela externa ao serviço. O fato de quase a metade dos antibacterianos dispensados ter sido para atender a clientela externa ao serviço aponta para as dificuldades de programação e distribuição desse tipo de medicamento.

Apesar das limitações impostas pela ausência de dados da especialidade do prescritor, pode-se verificar que o generalista vem assumindo um papel importante na prescrição ambulatorial de antibacterianos. Esse aspecto merece destaque em virtude do recém-implantado Programa Saúde da Família em Belo Horizonte, mostrando sua rápida disseminação e o papel que estes profissionais passam a ter no contexto da prescrição de medicamentos.

A Organização Mundial da Saúde propõe indicadores para o estudo da prescrição, por meio dos quais é possível descrever o uso local de medicamentos e acompanhar sua evolução temporal. Alguns desses indicadores foram avaliados neste estudo, entre eles o número médio de medicamentos por prescrição de antibacteriano, que foi de 2,4; deve-se ressaltar, porém, que cerca de $30 \%$ das prescrições continham três medicamentos. Fegadolli et al. 16, em estudo de prescrições pediátricas em município do interior de São Paulo, observaram um número médio de 2,6 medicamentos por receita. Jankovic et al. 14 verificaram que esse número variou de 1,0 a 2,8 em unidades de saúde da Sérvia. Berquó et al. 17, em um estudo de base populacional do uso de antimicrobianos na zona urbana de Pelotas, Rio
Grande do Sul, Brasil, referiram que cerca de $20 \%$ da população amostrada relatou o uso de outros medicamentos juntamente com o antimicrobiano. Esses dados indicam que os antibacterianos, na sua grande maioria, são utilizados com pelo menos mais um medicamento, o que parece estar relacionado ao tratamento sintomático das infecções.

Foram observadas, em um pequeno percentual, associações de antibacterianos. Tais associações só se justificam em situações clínicas específicas, pouco comuns na atenção primária. Contudo, ainda que em baixo número, estas associações devem ser pesquisadas para uma avaliação mais rigorosa de sua adequação e racionalidade.

A amoxicilina foi o antimicrobiano mais prescrito, confirmando os dados da SMSA/BH, onde este medicamento tem sido o mais consumido nos últimos anos. Esse perfil de utilização é esperado por tratar-se de antimicrobiano de amplo espectro e baixa toxicidade, constituindo tratamento de primeira escolha para vários quadros infecciosos. Além disso, a grande experiência de uso clínico desse fármaco confere maior segurança ao prescrevê-lo. O seu extenso uso na atenção primária tem sido descrito por outros autores 1,17 .

A benzilpenicilina benzatina foi o segundo antibacteriano mais prescrito, todavia esse medicamento apresenta várias desvantagens. É um injetável de depósito, mantém concentrações plasmáticas baixas, é de liberação lenta, não controlada, não tem dose de ataque, pode causar lesão ou dor no local da aplicação e ainda tem o risco de provocar reação anafilática. As características de administração em dose única para a maioria das doenças, além de seu baixo custo, são argumentos que reforçam sua utilização em atenção primária e o seu aparecimento como o segundo mais prescrito nas unidades da GERSA-CS

A eritromicina é um medicamento de uso mais restrito na atenção primária em face das opções disponíveis, o que pode explicar seu aparecimento em baixo percentual. Já a associação sulfametoxazol/trimetoprim constitui terapêutica de primeira escolha para tratamento das infecções do trato urinário e opção em outras doenças na atenção primária 13,18

Berquó et al. 17 observaram que as penicilinas, principalmente a amoxicilina, e o sulfametoxazol/trimetoprim corresponderam aos medicamentos de maior prevalência de uso, independentemente da faixa etária. Ressalta-se aqui que, mesmo em pequeno percentual, foram observadas prescrições de medicamentos de segunda geração, como a norfloxacina e a amoxicilina/clavulanato, cefalosporinas, outras 
fluoroquinolonas e a azitromicina. Tais medicamentos não são normalmente de escolha para tratamento das infecções comunitárias, devendo ser reservados para os casos em que sua indicação seja realmente necessária. O baixo percentual de prescrição de medicamentos de segunda geração, observado neste estudo, tem significado limitado, porque as receitas retidas são apenas aquelas que continham algum medicamento disponível na SMSA/BH. Como a maioria dos antibacterianos de segunda geração não é padronizada pelo serviço, as suas prescrições não ficaram retidas, exceto quando algum medicamento disponível na SMSA/BH foi prescrito junto ao antimicrobiano. Contudo, sabendo-se que os antimicrobianos de segunda geração são reservados para situações específicas, não usuais na atenção primária, e que a SMSA/BH tem feito um grande esforço para divulgar entre os prescritores a lista de medicamentos padronizados, acredita-se que as receitas internas retidas sejam representativas do total de antimicrobianos prescritos no âmbito da GERSA-CS.

O uso racional do medicamento tem como aspecto fundamental a orientação formal sobre como utilizá-lo, que deve ser impressa na prescrição e explicada verbalmente, particularmente para antibacterianos, cujo uso inadequado pode estar relacionado ao desenvolvimento de resistência. A presença de dados imprescindíveis, como a posologia e a duração do tratamento, é essencial para a dispensação e adesão do paciente.

Observa-se que os prescritores demonstraram uma preocupação particular com o registro da dose, presente em mais de $99 \%$ das receitas. Entretanto, parecem não atentar para a necessidade dos demais dados técnicos, importantes até para a própria dispensação do medicamento. Destaca-se, nesse contexto, uma ausência elevada do registro de informações essenciais para a dispensação e o uso dos antibacterianos, como a apresentação e a via de administração desses medicamentos. Observa-se particularmente a ausência do tempo de tratamento em cerca de $10 \%$ das receitas. Quando se verifica que os dois antibacterianos mais prescritos pertencem à classe dos $\beta$-lactâmicos, deve-se considerar que, de acordo com Burgess 19, para essa classe o pico de concentração não tem tanta importância, mas, sim, a duração do tempo em que a concentração é mantida acima da concentração inibitória mínima, sendo essencial atentar para a posologia e duração do tratamento.

Deve-se considerar que, diante da gravidade da disseminação de cepas resistentes, a ausência da definição de tempo de tratamento é um erro grave cujas conseqüências comprometem todo o esforço do serviço público de saúde na disponibilização dos antibacterianos. Além disso, a ausência desse item impossibilita a dispensação. A existência de cerca de $10 \%$ das prescrições sem definição do tempo de tratamento deixa dúvidas quanto à quantidade total dispensada pelo serviço e quanto ao tempo de utilização pelo paciente. Este fato demonstra a fragilidade do sistema de controle da dispensação de medicamentos na SMSA/BH, apontando a necessidade de treinamento dirigido aos atendentes e de auditorias sistemáticas no serviço.

A avaliação dos intervalos posológicos prescritos foi utilizada como uma forma de estimar a adequação das prescrições aos tratamentos preconizados pela literatura, para as infecções mais comumente atendidas na atenção primária. Foram observados intervalos posológicos atípicos para os cinco antibacterianos mais prescritos, com destaque para o sulfametoxazol/trimetoprim. Intervalos menores que 12 horas para o sulfametoxazol/trimetoprim podem, eventualmente, ser utilizados, mas raramente isso se aplica como uma abordagem inicial nas infecções comunitárias.

Essa mesma abordagem inicial, utilizada na avaliação da duração do tratamento, mostrou uma grande variabilidade nos tempos de tratamentos adotados. Intervalos inferiores a sete dias não são preconizados pela SMSA/BH para tratamento das infecções na atenção primária. Embora protocolos de curta duração possam ser efetivos em algumas situações clínicas específicas, sua adoção exige o acompanhamento da evolução do processo infeccioso, que nem sempre é possível no serviço público ambulatorial. A adoção de tratamentos por tempo inferior aos preconizados pode implicar falhas terapêuticas e favorecer o desenvolvimento de resistência bacteriana. Por outro lado, intervalos maiores que os recomendados na literatura não aumentam a efetividade do tratamento e podem acarretar risco potencial de toxicidade, além de elevar os custos da terapia. Vale ressaltar que situações particulares de tratamentos já iniciados não foram avaliadas, mas acredita-se que devam constituir casos isolados e, portanto, não comprometam o resultado observado.

Quando os resultados são comparados entre grupos de prescrições internas e externas, observa-se que, embora para algumas variáveis tenha havido diferença significativa entre eles, no geral, a qualidade da prescrição é similar. Ainda que a SMSA/BH desenvolva trabalhos de divulgação de informações terapêuticas, da padronização de tratamentos e medicamentos, as prescrições internas não apresentaram melhores resultados do que as externas. Nem mesmo para a prescrição 
pelo nome genérico, que é obrigatória no âmbito do SUS. Essa similaridade na qualidade da prescrição demonstra que as medidas normativas e informativas adotadas pela SMSA/BH não têm permitido atingir o objetivo de garantir melhorias na qualidade da prescrição.

Ressaltam-se também as falhas no processo de dispensação, apontando a necessidade de treinamento continuado dos atendentes e de monitoramento da adesão às medidas normativas preconizadas pela SMSA-BH.

Éimportante destacar que qualquer avaliação de qualidade dentro das práticas em saúde deve visar ao bem-estar do paciente, que é o foco da atenção dos profissionais. Normalmente, a avaliação e o monitoramento por meio de indicadores permitem detectar e corrigir falhas que possam comprometer a qualidade do serviço. Sendo assim, o presente estudo permitiu observar pontos vulneráveis da prescrição e dispensação de antimicrobianos, e tais vulnerabilidades devem ser objeto de análise e intervenções direcionadas para correção dos problemas detectados.

\section{Conclusões}

Os estudos de prescrição de antibacterianos são úteis por serem metodologicamente simples, permitindo sinalizações acerca do uso adequado dessa classe terapêutica. No entanto, tais estudos são limitados, não atingindo alguns dos principais aspectos do uso inadequado, que são a indicação e seleção do antimicrobiano em face do diagnóstico. Assim, os problemas aqui apontados representam uma primeira abordagem com ênfase nos aspectos mais elementares da questão os aspectos formais da prescrição e dispensação de antibacterianos. Destaca-se a necessidade de investigações mais aprofundadas, constituindo auditorias para uma avaliação mais abrangente do uso de antibacterianos nos serviços públicos de saúde.
Apesar das limitações no que se refere à amplitude da questão do uso de antimicrobianos, este estudo permitiu verificar que o padrão de prescrição de antibacterianos dispensados na GERSA-CS e SMSA/BH é similar a outros descritos na literatura, mantendo coerência com o perfil de atendimento da atenção primária. Contudo, o fato de a qualidade da prescrição ser similar entre prescritores externos e internos à SMSA/ $\mathrm{BH}$ aponta para falhas na adoção dos processos normativos instituídos pela SMSA/BH, no que se refere tanto à prescrição quanto à dispensação de antimicrobianos. Pode-se destacar, ainda, a ausência do registro na prescrição da duração do tratamento e a variabilidade nos períodos de tratamento instituídos como os principais problemas detectados. Nesse sentido, este estudo contribuiu para demonstrar a necessidade não só de ampliar o alcance das medidas informativas e regulatórias, como também de avaliar e melhorar outros aspectos que possam estar influenciando o processo, como falta de infraestrutura e recursos humanos qualificados, em número adequado.

É importante ressaltar que, além das conseqüências administrativas e econômicas desencadeadas pelos problemas evidenciados com relação à prescrição e à dispensação de antimicrobianos, existe uma questão social e outra sanitária envolvidas. No primeiro aspecto, os problemas observados na prescrição podem comprometer o acesso do paciente ao medicamento ou ao seu uso adequado. Como resultado, têm-se desperdícios, falência terapêutica e tratamentos inefetivos. A questão sanitária é decorrente da inadequação de doses, posologia ou tempo de tratamento, possibilitando o recrudescimento da infecção e desenvolvimento de resistência microbiana. Nesse contexto, fica claro que não basta apenas o diagnóstico preciso e a seleção adequada do antimicrobiano se a prescrição não for rigorosamente elaborada e completa em todos os seus aspectos. 


\section{Resumo}

O uso abusivo de antimicrobianos constitui um grave problema de saúde pública e tem suscitado discussões acerca da racionalidade de sua utilização. O objetivo deste trabalho foi investigar a prescrição de antimicrobianos de uso sistêmico em unidades de atenção primária da Gerência de Saúde Centro Sul da Secretaria Municipal de Saúde de Belo Horizonte, Minas Gerais, Brasil. Trata-se de um estudo transversal de avaliação das prescrições atendidas durante o mês março de 2002, utilizando-se indicadores da Organização Mundial da Saúde e do uso de antimicrobianos. Constatou-se que cerca de $20 \%$ das receitas continham pelo menos um antimicrobiano de uso sistêmico. A amoxicilina foi o antibacteriano mais prescrito, seguido pela benzilpenicilina benzatina. Observaram-se indices de presença de dados técnicos (dose, intervalo entre doses, via de administração e tempo de tratamento) nas prescrições variando de 23,6\% a 99,6\%. Em cerca de $10 \%$ das receitas, não havia definição do tempo de tratamento e houve uma grande variabilidade na duração deste. Este estudo evidenciou a necessidade de implementação de medidas informativas e normativas que visem a melhorar a qualidade da prescrição de antimicrobianos no nível ambulatorial.

Agentes Antibacterianos; Prescrição de Medicamentos; Atenção Primária à Saúde

\section{Colaboradores}

P. M. Abrantes colaborou na revisão da literatura, elaboração da metodologia, análise dos resultados e redação do artigo final. S. M. S. Magalhães participou da revisão da literatura, revisão da análise dos resultados e redação do artigo final. F. A. Acúrcio elaborou a metodologia. E. Sakurai contribuiu na análise estatística dos resultados.

\section{Referências}

1. Lirola MAG, Barrera JC, Garcia JMI, Asensio AR. La calidad de la prescripción de antibacterianos em un distrito de atención primaria. Evolución 19941995. Aten Primaria 1997; 19:487-92.

2. Vergeles-Blanca JM, Aguillar JAF, Bermejo RH, Retamosa FE, Torres JAC, Buitrago F. Calidad y características de la prescripción de antibióticos em un servicio hospitalario de urgencias. Rev Esp Salud Pública 1998; 72:111-8.

3. Fijn R, Chow MC, Schuur PMH, Berg DJV, Brouwers JRBJ. Multicentre evaluation of prescribing concurrence with anti-infective guidelines: epidemiological assessment of indicators. Pharmacoepidemiol Drug Saf 2002; 11:361-72.

4. Wolff MJ. Use and misuse of antibiotics in Latin America. Clin Infect Dis 1993; 17 Suppl 2:S346-51.

5. Arnold SR, Allen UD, Al-Zahrani M, Tan DHS, Wang EEL. Antibiotic prescribing by pediatricians for respiratory tract infection in children. Clin Infect Dis 1999; 29:312-7.
6. Dosh SA, Hickner JM, Mainots AG, Ebell MH. Predictors of antibiotic prescribing for nonspecific upper respiratory infections, acute bronchitis, and acute sinusitis. J Fam Pract 2000; 49:407-14.

7. Davey PJ, Bax RP, Newey J, Reeves D, Rutherford $\mathrm{D}$, Slack R, et al. Growth in the use of antibiotics in the community in England and Scotland in 198093. BMJ 1996; 312:613.

8. Acúrcio FA, Perini E, Magalhães SMS, Vieira Terceiro LG, Abrantes PM, Vale SA, et al. Analysis of medical prescriptions dispensed at health centers in Belo Horizonte, Minas Gerais, Brazil. Cad Saúde Pública 2004; 20:72-9.

9. Shab SN, Aslam M, Avery AF. A survey of prescription errors in general practice. The Pharmaceutical Journal 2001; 267:860-2.

10. Prefeitura Municipal de Belo Horizonte. BH da gente - conselhos regionais populares. Belo Horizonte: Prefeitura Municipal de Belo Horizonte; 2001. 
11. WHO Collaborating Centre for Drug Statistics Methodology. Anatomical therapeutic chemical (ATC) classification index with defined daily doses (DDDs). Oslo: World Health Organization; 2000.

12. The United States Pharmacopeial Convention. Drug information for the health care professional (USP-DI). 22nd Ed. Massachusetts: The United States Pharmacopeial Convention; 2002.

13. Núcleo de Assistência Farmacêutica, Escola Nacional de Saúde Pública. Fundamentos farmacológicos-clínicos dos medicamentos de uso corrente 2002. Rio de Janeiro: Escola Nacional de Saúde Pública; 2002.

14. Jankovic SM, Vasic LM, Maksimovic MR, Cupurdija VB, Kostic IR, Kovacevic ZN. An analysis of drug use indicators in primary care health facilities operating in the city of Kragujevac. http://priory. com/fam/kosovo.htm (acessado em Ago/2002).
15. Pascual-Salcedo MA, Caamaño MB, Fernández MVF, Tanco MG. Consumo extrahospitalario de antiinfecciosos en dosis diaria definida por mil habitantes y dia. Aten Primaria 1998; 18:357-61.

16. Fegadolli C, Mendes IJM, Simões MJS. Avaliação da prescrição médica em pediatria, baseada nos indicadores do uso de medicamentos selecionados pela OMS em município do interior do Estado de São Paulo. Rev Ciênc Farm 2002; 23:239-54.

17. Berquó LS, Barros AJD, Lima RC, Bertoldi AD. Utilização de antimicrobianos em uma população urbana. RevSaúde Pública 2004; 38:239-46.

18. Ministério da Saúde. Manual de condutas médicas: Programa Saúde da Família. São Paulo: Ministério da Saúde; 2001.

19. Burgess DS. Pharmacodynamic principles of antimicrobial therapy in the prevention of resistance. Chest 1999; 115 (3 Suppl):19S-23S.

Recebido em 03/Nov/2005

Aprovado em 02/Fev/2006 